\title{
The Ruby UCSC API: accessing the UCSC genome database using Ruby
}

\author{
Hiroyuki Mishima ${ }^{1 *}$, Jan Aerts ${ }^{2,3}$, Toshiaki Katayama ${ }^{4}$, Raoul J P Bonnal ${ }^{5}$ and Koh-ichiro Yoshiura ${ }^{1}$
}

\begin{abstract}
Background: The University of California, Santa Cruz (UCSC) genome database is among the most used sources of genomic annotation in human and other organisms. The database offers an excellent web-based graphical user interface (the UCSC genome browser) and several means for programmatic queries. A simple application programming interface (API) in a scripting language aimed at the biologist was however not yet available. Here, we present the Ruby UCSC API, a library to access the UCSC genome database using Ruby.

Results: The API is designed as a BioRuby plug-in and built on the ActiveRecord 3 framework for the object-relational mapping, making writing SQL statements unnecessary. The current version of the API supports databases of all organisms in the UCSC genome database including human, mammals, vertebrates, deuterostomes, insects, nematodes, and yeast.

The API uses the bin index-if available-when querying for genomic intervals. The API also supports genomic sequence queries using locally downloaded *.2bit files that are not stored in the official MySQL database. The API is implemented in pure Ruby and is therefore available in different environments and with different Ruby interpreters (including JRuby).

Conclusions: Assisted by the straightforward object-oriented design of Ruby and ActiveRecord, the Ruby UCSC API will facilitate biologists to query the UCSC genome database programmatically. The API is available through the RubyGem system. Source code and documentation are available at https://github.com/misshie/bioruby-ucsc-api/ under the Ruby license. Feedback and help is provided via the website at http://rubyucscapi.userecho.com/.
\end{abstract}

\section{Background}

The University of California, Santa Cruz (UCSC) genome database [1] is one of the most common gateways to access genomic sequence and annotation data of humans and other organisms. Besides a web-based genome browser [2], the database is programmatically accessible through three interfaces: the official commandline tools and libraries [3,4], the Distributed Annotation System (DAS) [5] server, and direct access to a public MySQL database server. UCSC's official tools consist of command-line executables and API libraries written in the $C$ language. The $C$ API widely supports the functionality of the database with good performance. These tools and libraries are available at the Kent source tree $[3,6]$. The UCSC DAS server, which supports previous

\footnotetext{
* Correspondence: hmishima@nagasaki-u.ac.jp

${ }^{1}$ Department of Human Genetics, Nagasaki University Graduate School of Biomedical Sciences, 1-12-4 Sakamoto, Nagasaki, Nagasaki 852-8523, Japan Full list of author information is available at the end of the article
}

DAS version 0.95 , offers a simple interface for programmatic access to the database. However, it has a limitation in supported types of annotations and has disadvantages in its performance. The public MySQL server, finally, offers access to almost the same up-to-date database for the genome browser but requires the user to program raw SQL statements. Given the pervasive use of scripting languages in this field of research, there is a significant demand for simple APIs that allow construction of automated queries in these languages. In particular, the Ruby programming language has been widely adopted in the bioinformatics domain $[7,8]$. Libraries including BioRuby [9] and the Ruby Ensembl API [10] have shown the value of database APIs for Ruby.

Here, we describe the Ruby UCSC API, an API to query the UCSC genome database.

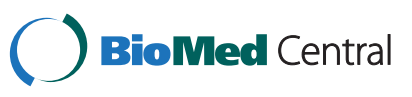

(c) 2012 Mishima et al.; licensee BioMed Central Ltd. This is an Open Access article distributed under the terms of the Creative Commons Attribution License (http://creativecommons.org/licenses/by/2.0), which permits unrestricted use, distribution, and reproduction in any medium, provided the original work is properly cited. 


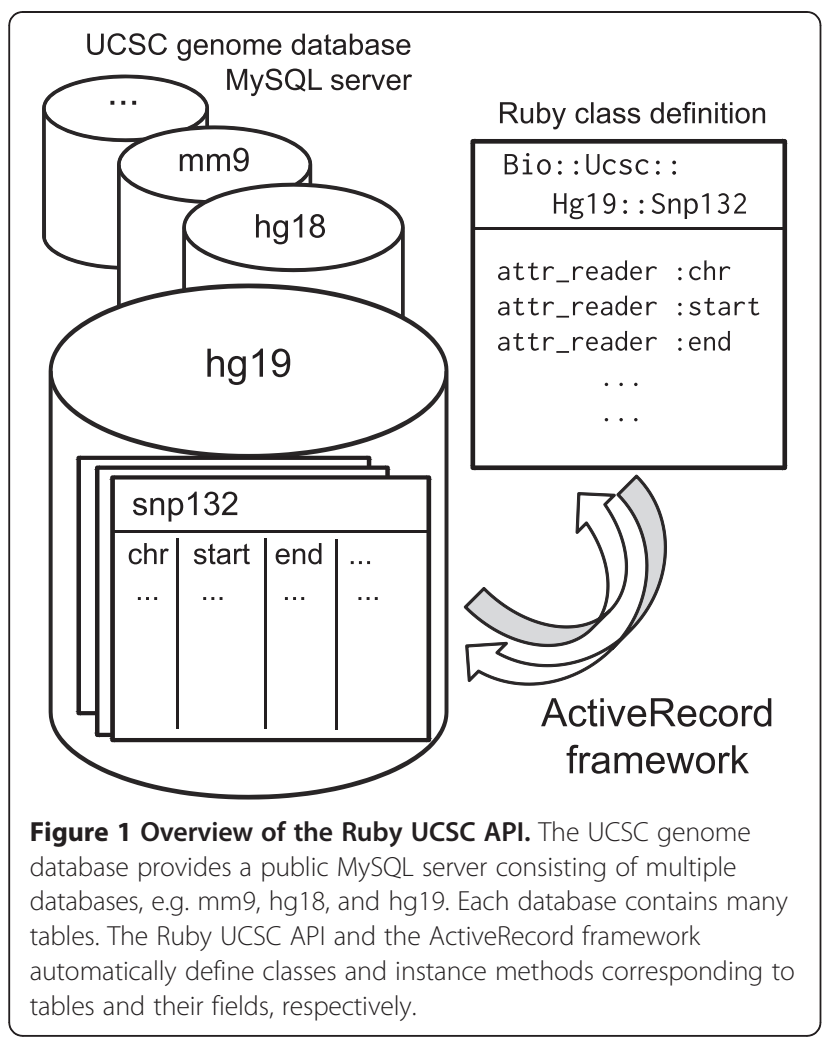

\section{Implementation}

\section{Object-relational mapping}

The Ruby UCSC API is based on the ActiveRecord 3 framework-a component of Ruby on Rails [11] - for the object-relational mapping (Figure 1). A database in the UCSC genome database is represented as a module under the Bio::Ucsc name space and a table in the database is represented as a class (subclass of the ActiveRecord::Base class) under the database module. For example, the "snp132" table in the human genome assembly "hg19" database is referred to as Bio::Ucsc:: Hg19::Snp132. Query APIs to a table are automatically defined from the database schema as class methods following the ActiveRecord's method naming convention. For example, if the "snp132" table has a field (column) "name", the Snp132.find_by_name method is readily available. Records (rows) are instances of the corresponding table class, for which values of any field can be obtained.

\section{Dynamic class definition}

The UCSC database is optimized to serve the genome browser, resulting in a very large number of tables (about 41,840 tables as MySQL *.MYD files) for which the API has to provide access. Furthermore, these database components are updated frequently. Static definitions of many table classes would make API code maintenance difficult. Therefore, we employed dynamic class definition in the Ruby UCSC API. When a table is referred to for the first time, the API fetches the database schema of that table to determine the data types and then creates an appropriate Ruby class for that table. This lazy generation of the classes also contributes to accelerate the initialization of this API when compared to having static classes for thousands of tables.

\section{Supporting auxiliary flat files}

A subset of the UCSC genome database, including genome sequences, is not stored in the MySQL database but needs to be downloaded locally for access. The Ruby UCSC API offers methods to access these downloaded genome sequences (".2bit files).

\section{Dependencies and environment}

The Ruby UCSC API depends on ActiveRecord 3 and is designed as a BioRuby plugin using the Biogem system $[12,13]$, which organizes RubyGems packages and their dependencies for the BioRuby library.

The Ruby UCSC API is written purely in Ruby. This increases the compatibility of the API for various operating systems and implementations of the Ruby interpreter. The API currently supports different Ruby interpreters including Ruby version 1.9.2 or later, Ruby version 1.8 .7 or later, and JRuby 1.6 .3 or later.

\section{Results and discussion}

Features and usage

Figure 2 shows examples of the Ruby UCSC API in use.

\section{Database connection}

After loading the Ruby UCSC API library (line 2), a connection to a database can be established by the 'connect' method (line 6). While the default connection is made against the UCSC public MySQL server, alternative full or partial mirror servers can be used as well (line 9-11). The API can connect to multiple databases simultaneously.

\section{Table query}

Users can query the database by series of "find" class methods which are dynamically defined for each table class by the ActiveRecord. First of all, find_by_[fieldname] and find_all_by_[field-name] class methods retrieve the first or all matching records, respectively. Example queries for the "name" field are shown in line 13. Multiple conditions joined by the _and_operator are also accepted (line 29). According to the ActiveRecord's convention, values of the other fields in a retrieved record can be referred to by using instance methods denoted by the field names (line 14). 


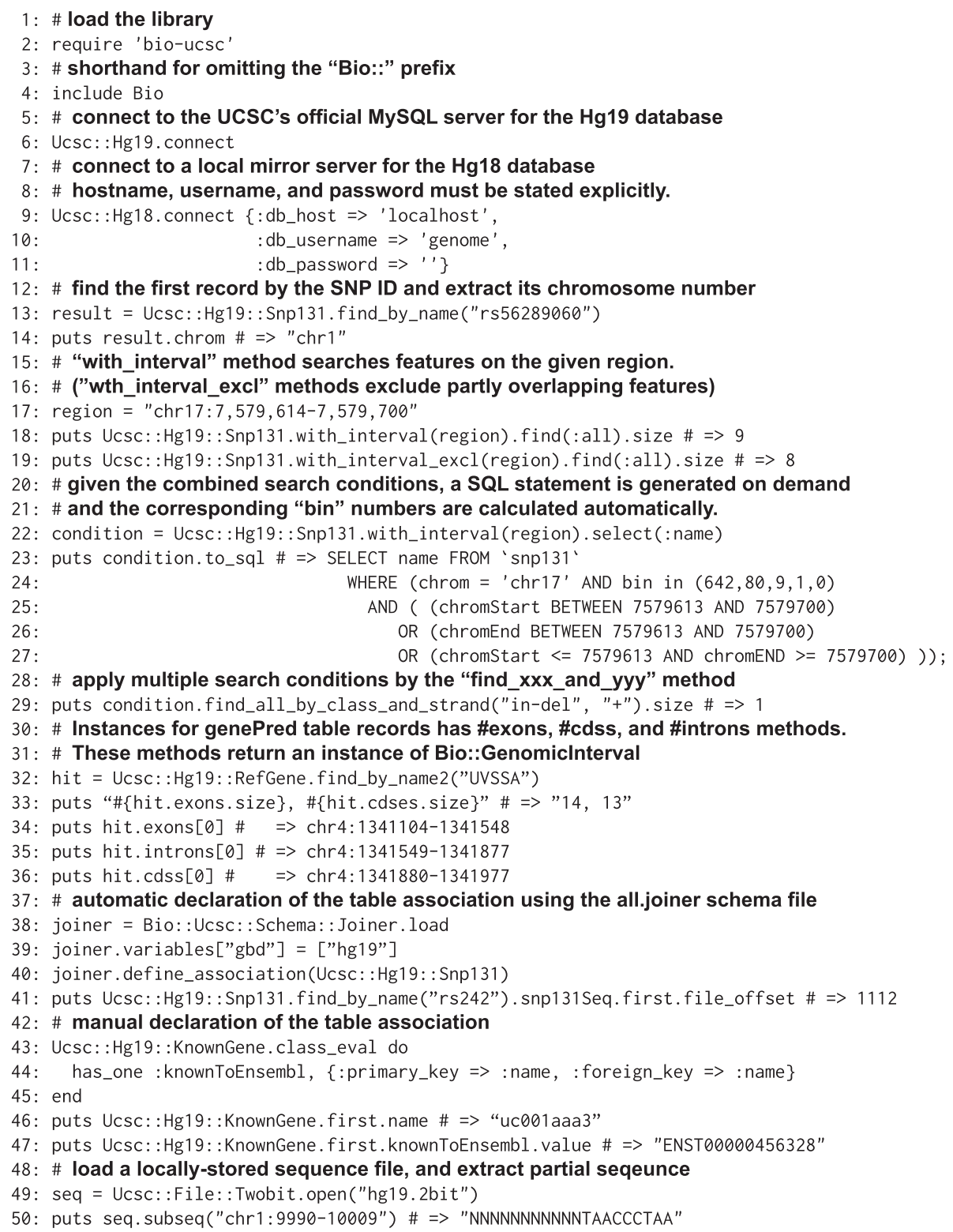

Figure 2 Example script using Ruby UCSC API.

\section{Query by genomic intervals}

A genomic interval can be expressed by a string like "chr1:123,456-456,789" as used in the graphical web interface of the UCSC Genome Browser (line 17). An interval query condition is passed by the with_interval method (line 18-19). This method automatically absorbs the difference of genomic coordinate conventions between intuitive 1-based coordinates and database internal 0-based coding system (compare line 17 and 25-27). The with_interval method allows retrieving all features that are overlapping with the given interval (line 18). Instead, the with_interval_excl method only returns features that lie completely within the region and features partially overlapping with the region are excluded (line 19).

\section{Bin indexing system}

To achieve high query performance for large tables, the UCSC database uses a bin indexing system [6]. In this system, genomic positions in a chromosome are separated into hierarchies of bins that are sized into 512Mbase, 64Mbase, 8Mbase, 1Mbase and $128 \mathrm{kbase}$. Any annotation in a genomic interval is stored in the minimum sized bin that encompasses the whole interval. For a genomic interval query, if the target table has a "bin" field, the API automatically calculates a list of bins 
that potentially contain annotations for the interval and applies the list to generate an SQL statement to narrow the target record. This is a key feature of the API because multiple queries for genomic intervals without using the bin index take excessive times, especially for large tables such as dbSNP.

\section{Building SQL statements}

Methods to specify search conditions, such as with_interval, select, where, order, limit and group, can be combined by chaining (line 22-27). When a find method or one of the methods to access arrays (such as find_all, first and []) is called for the condition, the constructed SQL query is executed and the results are returned (line 29).

Methods to access information of exons, CDSs, and introns Instances of "genePred" table classes, such as RefGene, EnsGene, and KnownGene, have exons, cdss, and introns methods. These methods return arrays of Bio:: GenomicInterval objects sorted according to the gene strand (line 32-36).

\section{Table association}

The joiner schema file describes the links between the tables of the UCSC genome database. The Bio::Ucsc:: Schema:::Joiner.load class method takes an URI of the schema file. If the URI is not given, UCSC's all.joiner file [14] is used (line 38). The format of the joiner file is documented in the Kent source tree [15]. Variables in the joiner file can be overwritten. For example, overwriting the gbd variable that stores whole databases can restrict databases used for the link search (line 39). The define_association method takes a table class and defines all the associations of given table (line 40). Unconnected databases and undefined tables are ignored during definition. Linked results are always returned as an array (line
41). The table association also can be defined manually. When a record in a table can be joined with a record of another table by sharing the same value (foreign key), the has_one / has_many methods are used to declare the association (line 43-45). Once the table association is declared, a table can refer to the associated table using a method of its record object (line 46-47).

\section{Retrieval of genomic sequences}

Extraction of genomic sequences in the given genomic intervals is a frequent task. The UCSC genome database does not store the genomic sequences in the MySQL databases. Instead, they provide the sequences as ".2bit files. These files are usually processed by UCSC's tools written in C. To improve the compatibility, we implemented the same functionalities in Ruby. With the Bio:: Ucsc::File::Twobit class, ".2bit files are interpreted in Ruby and subsequences can be extracted by the subseq method (line 49-50).

\section{Current limitations}

The current version of Ruby UCSC API uses information of the joiner schema file to find table associations. The all.joiner file, however, describes additional information of including which tables are chromosome- rather than genome-based, field values that have to be transformed to define table associations, and tables with exceptional structures. In future versions, the API will use this information to make user scripts simpler and to follow database structure updates immediately. So far, manual definition of table associations still has an advantage in performance by minimizing table association definitions, especially in some tables that have complicated associations.

For some tables including subsets of the Encyclopedia of DNA Elements (ENCODE) [16], the actual data are not stored in the MySQL database itself but are stored

Table 1 Supported databases

\begin{tabular}{|c|c|}
\hline Clade/organism & Databases \\
\hline human & $\mathrm{Hg} 19, \mathrm{Hg} 18$ \\
\hline mammals & $\begin{array}{l}\text { chimp (PanTro3), orangutan (PonAbe2), rhesus (RheMac2), marmoset (CalJac3), mouse (Mm9), rat (Rn4), guinea pig (CavPor3), } \\
\text { rabbit (OryCun2), cat (FelCat4), panda (AilMel1), dog (CanFam2), horse (EquCab2), pig (SusScr2), sheep (OviAri1), cow (BosTau4), } \\
\text { elephant (LoxAfr3), opossum (MonDom5), platypus (OrnAna1) }\end{array}$ \\
\hline vertebrates & $\begin{array}{l}\text { chicken (GalGal3), zebra finch (TaeGut1), lizard (AnoCar2), X. tropicalis (XenTro2), zebrafish (DanRer7), tetraodon (TetNig2), fugu } \\
\text { (Fr2), stickleback (GasAcu1), medaka (OryLat2), lamprey (PetMar1) }\end{array}$ \\
\hline deuterostomes & lancelet (BraFlo1), sea squirt (Ci2), sea urchin (StrPur2) \\
\hline insects & $\begin{array}{l}\text { D.melanogaster (Dm3), D.simulans (DroSim1), D.sechellia (DroSec1), D.yakuba (DroYak2), D.erecta (DroEre1), D.ananassae (DroAna2), } \\
\text { D.pseudoobscura (Dp3), D.persimilis (DroPer1), D.virilis (DroVir2), D.mojavensis (DroMoj2), D.grimshawi (DroGri1), Anopheles } \\
\text { mosquito (AnoGam1), honey bee (ApiMel2) }\end{array}$ \\
\hline nematodes & C.elegans (Ce6), C.brenneri (CaePb3), C.briggsae (Cb3), C.remanei (CaeRem3), C.japonica (CaeJap1), P.pacificus (PriPac1) \\
\hline others & sea hare (AplCal1), yeast (SacCer2) \\
\hline $\begin{array}{l}\text { common } \\
\text { databases }\end{array}$ & Go, HgFixed, Proteome, UniProt, VisiGene \\
\hline
\end{tabular}


as references to BigWig, BigBed [17] and BAM [18] files. BigWig and BigBed can be accessed by the UCSC tools in C. BAM files can be processed by third-party tools such as Samtools [18], and Picard [19]. To date, the Ruby UCSC API does not support these yet, however, users can use the bio-samtools BioRuby plugin [20] for these tasks.

\section{Existing UCSC APIs for scripting languages}

APIs for the UCSC genome database using scripting languages are still limited. For Perl, the Genoman module [21] offers interfaces to databases including the UCSC genome database. For Python, the Cruzdb library [22] offers an SQLAlchemy-based API for the UCSC genome database. The biggest advantage of Ruby UCSC API described here is that Ruby and the Active Record framework enable simplified query and retrieved record description. Moreover, the Ruby UCSC API does not depend on UCSC's command-line tools. This makes its installation easier and increases interoperability for various environments including a Java-based Ruby interpreter, JRuby.

\section{Conclusions}

UCSC's official executables and C libraries are the most comprehensive and fastest API for the UCSC genome database; however, APIs for scripting languages still have significant advantages for users because their concern is not only a runtime speed but also a total time required for the programming to obtain the results. The Ruby UCSC API offers effective productivity and can therefore have a significant impact in the field.

The Ruby UCSC API already supports all organisms in the UCSC genome database (Table 1). In future releases, more comprehensive supports for new organisms and older or updated genome assemblies will be added.

The Ruby UCSC API is freely available as a Rubygem package. Source code and documentations are also available at https://github.com/misshie/bioruby-ucsc-api/. Documentation and feedback are available at the UserEcho site at http://rubyucscapi.userecho.com/.

\section{Availability and requirements \\ Project name: The Ruby UCSC API \\ Project home page:}

https://github.com/misshie/bioruby-ucsc-api

Feedback and help: http://rubyucscapi.userecho.com/

Operation systems: Platform independent

Programming language: Ruby

Other requirements: Ruby interpreter (Ruby 1.8.7 or later, Ruby 1.9.2 or later, or JRuby 1.6.3 or later), and ActiveRecord (version 3.0.7 or later).

License: The Ruby License

Any restrictions to use by non-academics: none
Competing interests

The authors declare that they have no competing interests.

\section{Authors' contributions}

HM and JA conceived the project and implemented the API. TK, RJPB and KY reviewed the API design and implementation. HM, JA, TK, RJPB and KY wrote the manuscript. All authors read and approved the final manuscript.

\section{Acknowledgements}

HM is supported by Japan Society for the Promotion of Science, Grant-in-Aid for Young Scientists (B) [grant numbers 23791230]. JA is supported by SymBioSys II project funding [KUL PFV/10/016 SymBioSys], BioMaGNet [IUAP P6/25], the Research Council KU Leuven GOA MaNet, FP7-HEALTH CHeartED, and IBBT. KY is supported by grants from the Ministry of Health, Labour and Welfare; and the Uehara Memorial Foundation.

\section{Author details}

'Department of Human Genetics, Nagasaki University Graduate School of Biomedical Sciences, 1-12-4 Sakamoto, Nagasaki, Nagasaki 852-8523, Japan. ${ }^{2}$ Faculty of Engineering - ESAT/SCD, Leuven University, Kasteelpark Arenberg 10 - bus 2446, 3001, Leuven, Belgium. ${ }^{3}$ BBBT-KULeuven Future Health Department, Leuven, Belgium. ${ }^{4}$ Database Center for Life Science, Research Organization of Information and Systems, Faculty of Engineering Bldg. 12, The University of Tokyo, 2-11-16, Yayoi, Bunkyo-ku, Tokyo 113-0032, Japan. ${ }^{5}$ Integrative Biology Program, Fondazione Istituto Nazionale di Genetica Molecolare, via Francesco Sforza, 28-20122, Milan, Italy.

Received: 8 April 2012 Accepted: 17 September 2012

Published: 21 September 2012

\section{References}

1. Fujita PA, Rhead B, Zweig AS, Hinrichs AS, Karolchik D, Cline MS, Goldman M, Barber GP, Clawson H, Coelho A, Diekhans M, Dreszer TR, Giardine BM, Harte RA, Hillman-Jackson J, Hsu F, Kirkup V, Kuhn RM, Learned K, Li CH, Meyer LR, Pohl A, Raney BJ, Rosenbloom KR, Smith KE, Haussler D, Kent WJ: The UCSC Genome Browser database: update 2011. Nucleic Acids Res 2010, 39:D876-D882.

2. UCSC Genome Browser. http://genome.ucsc.edu/

3. Kent W: Kent source tree. http://hgdownload.cse.ucsc.edu/admin/jksrc.zip.

4. Schattner P: Automated Querying of Genome Databases. PLoS Comput Biol 2007, 3:e1.

5. Dowell R, Jokerst R, Day A, Eddy S, Stein L: The distributed annotation system. BMC Bioinforma 2001, 2:7.

6. Kent WJ, Sugnet CW, Furey TS, Roskin KM, Pringle TH, Zahler AM, Haussler D: The human genome browser at UCSC. Genome Res 2002, 12:996-1006.

7. Aerts J, Law A: An introduction to scripting in Ruby for biologists. BMC Bioinforma 2009, 10:221.

8. Mishima H, Sasaki K, Tanaka M, Tatebe O, Yoshiura K: Agile parallel bioinformatics workflow management using Pwrake. BMC Research Notes 2011, 4:331-338.

9. Goto N, Prins P, Nakao M, Bonnal R, Aerts J, Katayama T: BioRuby: Bioinformatics software for the Ruby programming language. Bioinformatics 2010, 26:2617-2619.

10. Strozzi F, Aerts J: A Ruby API to query the Ensembl database for genomic features. Bioinformatics 2011, 27:1013-1014.

11. Hansson DH, the Rails core team: Ruby on Rails. http://rubyonrails.org/.

12. Prins P: biogems.info. http://www.biogems.info/.

13. Bonnal RJP, Aerts J, Githinji G, Goto N, MacLean D, Miller CA, Mishima H, Pagani M, Ramirez-Gonzalez R, Smant G, Strozzi F, Syme R, Vos R, Wennblom TJ, Woodcroft BJ, Katayama T, Prins P: Biogem: an effective tool based approach for scaling up open source software development in bioinformatics. Bioinformatics 2012, 28:1035-1037.

14. UCSC Bioinformatics Group: all.joiner. http://genome-source.cse.ucsc.edu/ gitweb/?p=kent.git;a=blob_plain,f=src/hg/makeDb/schema/all.joiner; $\mathrm{hb}=\mathrm{HEAD}$.

15. UCSC Bioinformatics Group: The joiner file. http://genome-source.cse.ucsc. edu/gitweb/?p=kent.git;a=blob;f=src/hg/makeDb/schema/joiner.doc $\mathrm{hb}=\mathrm{HEAD}$.

16. The ENCODE Project Consortium: A user's guide to the Encyclopedia of DNA Elements (ENCODE). PLoS Biol 2011, 9:e1001046. 
17. Kent WJ, Zweig AS, Barber G, Hinrichs AS, Karolchik D: BigWig and BigBed: enabling browsing of large distributed data sets. Bioinformatics 2010, 26:2204-2207.

18. Li H, Handsaker B, Wysoker A, Fennell T, Ruan J, Homer N, Marth G, Abecasis G, Durbin R, 1000 Genome Project Data Processing Subgroup: The sequence alignment/map format and SAMtools. Bioinformatics 2009, 25:2078-2079.

19. Picard. http://picard.sourceforge.net/.

20. Ramirez-Gonzalez RH, Bonnal R, Caccamo M, MacLean D: Bio-samtools: Ruby bindings for SAMtools, a library for accessing BAM files containing high-throughput sequence alignments. Source Code for Biology and Medicine 2012, 7:6.

21. Engström P: Genoman. http://www.ebi.ac.uk/ engstrom/genoman/.

22. Pedersen B: Cruzdb. https://github.com/brentp/cruzdb.

doi:10.1186/1471-2105-13-240

Cite this article as: Mishima et al.: The Ruby UCSC API: accessing the UCSC genome database using Ruby. BMC Bioinformatics 2012 13:240.

\section{Submit your next manuscript to BioMed Central and take full advantage of:}

- Convenient online submission

- Thorough peer review

- No space constraints or color figure charges

- Immediate publication on acceptance

- Inclusion in PubMed, CAS, Scopus and Google Scholar

- Research which is freely available for redistribution 